\title{
PHYSIOLOGICAL STUDIES ON THE HEAVY METALS RESISTANCE MECHANISMS IN TOMATO PLANTS GROWING UNDER INTERCROPPING CONDITIONS.
}

\author{
A.M. Abd El-All, Esraa A. Morsy and Mervat E. Sorial \\ Botany Dept., Faculty of Agriculture, Menoufia University.
}

Received: May 27, 2021

Accepted: Jun. 23, 2021

\begin{abstract}
Pot experiments were conducted in the greenhouse of the Faculty of Agriculture in Shebin El-Kom, Menoufia University, during 2018 and 2019 seasons, to study the cultivation of (sensitive) tomato plants in a loading system with watercress plants (resistant) in a soil contaminated with heavy metals. Three levels of lead were used, which are 0 (control), 1000 and $2000 \mathrm{mg} / \mathrm{kg}$ of soil, and three levels of cadmium metal, which are 0 (control), 100 and $300 \mathrm{mg} / \mathrm{kg}$ of soil, each alone. Use of growth inducers as nanoparticles silicon and seaweed extract and its affects on tomato or watercress plants under heavy metals stress.
\end{abstract}

The characteristics of vegetative growth and some of the physiological and chemical characteristics such as photosynthetic pigments, water relations, activity of some antioxidant enzymes, $\mathrm{Pb}$ and $\mathrm{Cd}$ accumulation and some quantitative and qualitative characteristics of the yield were studied. The soil contamination with lead and cadmium minerals at all levels led to a decrease in all studied parameters. There was an increase in the accumulation of lead and cadmium metal within watercress plants by increasing the concentrations of lead and cadmium in the contaminated soil, and there was a decrease of the heavy metals residual in the polluted soil. Spraying with silicon and seaweed extract on tomato plants increased the vegetative growth characteristics, relative water content, plant pigments, yield and its components for tomato plants. While it led to a decrease in the concentration of the enzymatic activity of peroxidase and phenoloxidase. Also, increased the efficiency of watercress plants' accumulation of heavy metals. From the above, it is cleared that the use of hyperaccumulators plants (watercress) to accumulate heavy metals led to a decrease in the residual of the two heavy metals, lead and cadmium, in the contaminated soil, and this led to a better growth conditions for tomato plants grown in the intercropping system with watercress plants in soil contaminated with lead and cadmium.

Key words: Intercropping system, Tomato, Watercress, lead, Cadmium, Si and Seaweed.

\section{INTRODUCTION}

Heavy metal contamination is one of the most serious environmental problems limiting plant productivity and threatening human health. Amongst the substances that contribute anthropogenically to pollution of the biosphere, trace elements are one of the most toxic. Lead $(\mathrm{Pb})$ and cadmium $(\mathrm{Cd})$ are toxic metals of increasing environmental concern as they enter the food chain in increasingly significant amounts (Luptáková et al., 2002 \& Verma and Dubey, 2003).

In the world, contamination of agricultural lands associated with heavy metals is widespread, and heavy metals are one of the most prevalent factors that cause public health problems, and enter the body (i.e. crops grown on soil contaminated with heavy metals). A growing body of evidence indicates that 
soil organisms, critical to soil health and fertility, are sensitive to heavy metal stress (Dahlin et al. 1997) and that soil biodiversity is declining due to heavy metal contamination (Giller et al. 1998). In the future, moreover, plant extraction, which is the use of plants to extract heavy metals from contaminated soils, has emerged as a promising method for treating low to medium contamination soils (Salt et al. 1995). However, some ubiquitous heavy metals particularly lead; have limited plant uptake availability due to the complexity with hard soil fractions (Rieuwerts et al. 1998). Tomato (Solanum lycopersicum, family Solanaceae) is one of the most important vegetable crops in Egypt and other world countries for fresh consumption, industry processing and exportation.

Increasing the production and the quality of tomato fruits is very important objective to meet the higher human population demand.

Nanotechnology opens a large scope of novel application in the field of biotechnology and agricultural industries, because nanoparticles (NPs) have pore size, and particles morphology. Nanoparticles can serve as" magic bullets" containing herbicides, nano-pesticide fertilizers, or genes, which target specific cellular organelles in plant to release their content.

Nano Silicon (Si) fertilizer is known as
an ecologically compatible and environmental friendly technique to stimulate plant growth. Silicon was reported to reduce the hazard effects of various abiotic and biotic stresses including salt stress, metal toxicity, drought stress, radiation damage, various pests and diseases caused by both fungi and bacteria, nutrients imbalance, high temperature and freezing (Ma, 2004; Etesamy and Jeong 2018). Silicon has emerged as an important nutrient for a range of horticultural crops i.e., cucumber, strawberry and tomato (Ma, 2004).

Nano Seaweed has been used worldwide to increase plant growth and yield. Modern agriculture is searching for new biotechnologies that would allow for a reduction in the use of chemical inputs without negatively affecting crop yield or the farmers' income. In recent years, the use of natural seaweed as fertilizer has allowed for partial substitution of conventional synthetic fertilizer (Khan et al. 2009; Zodape et al. 2010). In addition, a number of commercial seaweed extract products are available for use in agriculture and horticulture and can be used as liquid extracts applied as foliar spray, soil drench, or in granular/powder form as soil conditioners and manure (Thirumaran et al. 2009, and Hussain et al. 2021).

Several techniques have been using for removing heavy metal contaminants from the environmental but these techniques have limitations such as high cost, long time, logistical problems and mechanical complexity. Phytoremediation can be used as an alternative solution for heavy metal remediation process because of its advantages as a cost-effective, efficient, environment- and eco-friendly technology based on the use of metalaccumulating plants. According to previous studies, several plants have a high potential as heavy metals bioaccumulator and can be used for phytoremediation process of heavy metals.

\section{MATERIALS AND METHODS}

The current research was conducted under controlled conditions of artificial pollution with lead and cadmium (heavy metals) in the greenhouse of the Agriculture Faculty, Menoufia University during the 2018 and 2019 seasons to study the effect of the $\mathrm{Pb}$ and $\mathrm{Cd}$ 
pollutants on tomato plants (sensitivity plant) which grown under intercropping system with watercress plants (hyperaccumulator plant) in soil polluted with different concentrations of the above mentioned heavy metals, and treated with foliar leaf applications as seaweeds and silicon in form of nanoparticles to investigate that, the phytoremediation (is a new method in which green plants are used to absorb or detoxify heavy metals), also study the absorption and transfer of lead and cadmium from the pollutant soil to both watercress and tomatoes, and the effect of plant sensors (foliar leaf) as seaweeds and silicon on phytoremediation method and intercropping system. Moreover, knowing the preference for cultivation on land contaminated with lead and cadmium by means of intercropping system or not.

Artificial pollution of the soil was done by adding lead salts in the form of $\mathrm{Pb}$ $\left(\mathrm{NO}_{3}\right)_{2}$ at concentrations of 0,1000 and $2000 \mathrm{mg}(\mathrm{Pb}) / \mathrm{kg}$ soil and cadmium in the form of $\mathrm{Cd} \quad \mathrm{Cl}_{2} . \quad 2 \mathrm{H}_{2} \mathrm{O}$ at concentrations of 0,100 and $300 \mathrm{mg}(\mathrm{Cd})$ / kg soil (Ernst, 1996).

Foliar leaf applications as Seaweeds $(0.5 \mathrm{ml} / \mathrm{l})$ nano Biostimulators contains [sea weeds $7 \%+$ salicylic acid $5 \%+$ proline $4 \%$ was obtained from Zhengzhou Zheng Shi Chemical Co., Ltd. China.And Silicon ((Si: $2 \mathrm{mM})$, nano Silicon $\left(\mathrm{SiO}_{2}\right)$ was obtained from Bio Nano Technology Com., Egypt. Foliar leaf applications were done at three times at 30,45 and 60 days from transplanting.

The experiment treatments were designed as complete randomizes design, tow-way analysis with four replications and included 15 treatments.

The seedlings of tomato plants and the watercress seeds aforementioned were obtained from the Horticulture Research Institute Agriculture Research Center in Cairo.
Ten seeds of watercress per pot were sown at 5th February in both seasons in pots $30 \mathrm{~cm}$ diameter, after 15 days the watercress seedlings were thinned to five uniform seedling, and three seedlings of tomato plants were transplanting after 20 days to the same pots which planted with the watercress seeds, each pot filled with $8 \mathrm{~kg}$ of clay sandy soil with ratio 2:1. The physical and chemical characteristics of experimental soil are shown in Table (1) according to Page (1982).

All pots were fertilized at the recommended rates of $\mathrm{N}, \mathrm{K}$ and $\mathrm{P}$ fertilizers according to the recommendations of agricultural ministry. After sowing, the pots were watered immediately by tap water through the experimental period whenever necessary maintain the soil water content at $65 \%$ of the water holding capacity. Weeds and best control as well as other agriculture practices were used whenever necessary.

\section{Sampling:}

Plant samples were taken at 60 days from transplanting to determine the following data:

\section{Growth characters:}

1.1. Root length $(\mathrm{cm})$.

1.2. Plant height (cm).

1.3. Number of leaves per plant.

1.4. Roots, Stem and Leaves dry weight.

1.5. Leaf area per plant $\left(\mathrm{cm}^{2}\right)$ using the dry weight method described by A.O.A.C. (1995).

\section{Water relations:}

2.1. Relative water content (RWC) was determined by using the method of Barrs and Weatherley (1962).

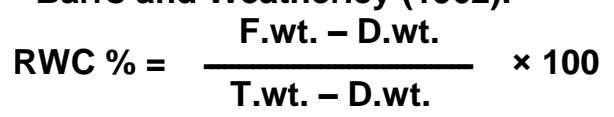

Where's, F.wt. = fresh weight of leaf $(g)$. (g). T.wt.= turgid (or saturated) weight

D.wt. = dry weight (g). 
A.M. Abd El-All, et al.,

2.2. Membrane leakage (ML) was

electrolyte conductivity (EC2) was determined as described by Sun et measured after boiling the plant al. (2006), and calculated as: samples for $15 \mathrm{~min}$.

$(E C 1 / E C 2) \times 100$. The initial

conductivity (EC1) and final

Table (1): Some physical and chemical properties of experimental soil:

\begin{tabular}{|c|c|}
\hline Properties & Value \\
\hline \multicolumn{2}{|c|}{ Physical analysis } \\
\hline Sand \% & 34.74 \\
\hline Silt \% & 15.86 \\
\hline Clay \% & 48.96 \\
\hline Texture & clay sandy \\
\hline \multicolumn{2}{|c|}{ Chemical analysis } \\
\hline $\mathbf{P H}$ & 7.64 \\
\hline O.M. \% & 0.68 \\
\hline $\mathrm{CaCO}_{3}$ & 1.51 \\
\hline Ec (mmhos/cm) & 1.9 \\
\hline \multicolumn{2}{|c|}{ Soluble ions (meq/100 g soil) } \\
\hline $\mathrm{HCO}_{3}$ & 0.59 \\
\hline $\mathrm{Cl}^{-}$ & 0.47 \\
\hline $\mathrm{SO}_{4}^{-2}$ & 0.77 \\
\hline $\mathrm{Na}^{+}$ & 0.53 \\
\hline $\mathrm{K}^{+}$ & 0.48 \\
\hline $\mathrm{Ca}^{+2}+\mathrm{Mg}^{+2}$ & 0.82 \\
\hline Total N (100) & 0.26 \\
\hline Avail. $\mathrm{P}\left(\mathrm{Mg} \cdot \mathrm{g}^{-1}\right)$ & 0.52 \\
\hline \multicolumn{2}{|c|}{ Heavy metals (mg/1000 g soil) } \\
\hline Lead & 0.98 \\
\hline Cadmium & 0.09 \\
\hline
\end{tabular}

\section{Photosynthetic pigments:}

According to the method described by Fadeel's, 1962 methods cited in A.O.A.C. (1995) then calculated as $\mathrm{mg} / \mathrm{g}$ D.wt.

\section{Determination of Antioxidant Enzymes activity:}

Peroxidase and phenoloxidase activity in optical density/g (O.D./g fresh weight after 2 and $45 \mathrm{~min}$ ), respectively were measured in the fresh leaves using the methods described by Fehrman and Dimond (1967) and Broesh (1954), respectively.

\section{Heavy metals accumulation:}

Accumulation of Heavy metals were determined by atomic absorption spectrophotometer (Model Perkin Elmer) and expressed as $\mu \mathrm{g} / \mathrm{g}^{-1}$ dry weight according to Cottenie et al., (1982).

\section{Yield and its components:}

At harvest (during the last month of tomato plants age), the following data were recorded:

Fruits number per plant, fruits weight per plant (g), fruit volume $\left(\mathrm{cm}^{3}\right)$ and heavy metal concentration in fruits were 
determined by using the above mentioned methods.

Vitamin C: Vitamin C content was determined in fruit juice using 2, 6dichlorophenolindo-phenol blue dye as mg ascorbic acid per $100 \mathrm{ml}$ Juice A.O.A.C. (1980), and expressed as $\mathrm{mg} / 100 \mathrm{ml}$ juice.

\section{Experimental design and statistical analysis:}

The experimental pots were arranged in a complete randomized block design with six replicates. Tow-way analysis of variance (ANOVA) was used to assess the significant difference in the heavy metal concentrations among different treatments, the standard statistical analysis following the proceeding described by Gomez and Gomez (1984) using the computer program of Costat Software (1985). The analyzed data then presented in tables.

\section{RESULTS AND DISCUSSION}

\section{Vegetative Growth characters:}

The data in Table (2) showed that, all concentrations of $\mathrm{Pb}$ and $\mathrm{Cd}$ caused $\mathrm{a}$ significant decrease in root length, plant height number of leaves, leaf area, of tomato plants. The concentrations of $\mathbf{C d}$ were more effect on tomato than $\mathrm{Pb}$ concentrations. The highest value of decrease was recorded at Cd level of $\mathbf{3 0 0}$ ppm. Results presented in Table (3) showed that, dry weight of root, stem, leaves and whole plants of tomato were significantly affected by different concentration of $\mathrm{Pb}$ and $\mathrm{Cd}$. The concentrations of $\mathrm{Cd} 100 \mathrm{ppm}$ were less harmful than $\mathrm{Cd}$ 300ppm.

From the results in Tables (2 and 3), it was observed that, the foliar application of seaweed and silicon (nanoparticles) caused a significant increase in previous growth parameters of tomato plants. The highest increases were recorded at treatment of SWE, at the first and the second seasons as compared with the untreated plants.

The interactions between heavy metals ( $\mathrm{Pb}$ and $\mathrm{Cd}$ ) and nanoparticles (SWE and Si) were observed in Tables (2 and 3 ) and citied that, all levels of $\mathrm{Pb}$ and caused a decrease in tomato growth parameters, but seaweed and silicon caused a recovering to a deleterious effects of heavy metals when spraying on tomato plants when compared with the control plants. These results were confirmed in the second season. These results were according to the findings by Shuyi et al. (2013) and who showed that, spraying silicon could promote growth of lettuce, and inhibit the absorption of $\mathrm{Cd}$ and $\mathrm{Pb}$ and decrease the content and accumulation of $\mathrm{Cd}$ and $\mathrm{Pb}$ in shoots and roots. These results are in agreement with the findings of Arun et al. (2014) and Hussain et al. (2021) who found that, the seeds treated with seaweed at different concentrations promote various parameters such as root length, shoot length and number of lateral roots of tomato plants. Sobkowiak (2016) reported that, the impact of $\mathrm{Cd}$ on the shoot length was not linearly related with the dose. Leaf number and leaf area had linear negative relationship with the dose of cadmium. Moreover, $C d$ reduced the growth of soybean in proportion to their doses. In addition, the $\mathrm{Si}$ is not an essential nutrient considered, but it is a beneficial element to mentian growth in stressfull environments. Facing the toxicity of $\mathrm{Cd}$ has also increased the resistance of various crops. In tomato, and cucumber plants, the concentration of $\mathrm{Cd}$ in the shoots and leaves decreased. (Zargar et al. 2019, Li et al. 2016, and Wu et al. 2015).

\section{Water relations \& Membrane integrity:}

Data presented in Table (4) showed that, there was a remarkable gradual decrease in RWC in leaves of tomato 
A.M. Abd El-All, et al.,

plants with concentrations of $\mathrm{Pb}$ and $\mathrm{Cd}$ compared with the control which reached about $21 \%$ on RWC with 300 ppm Cd at the first season. The highest increase in MI was recorded at $300 \mathrm{mg} / \mathrm{kg} \mathrm{Cd}$

Table (2): Effect of heavy metals, nanoparticles and their interactions on growth
characters of tomato plants at 60 days from transplanting during the growing

Table (2): Effect of heavy metals, nanoparticles and their interactions on growth
characters of tomato plants at 60 days from transplanting during the growing seasons of 2018 and 2019.

\begin{tabular}{|c|c|c|c|c|c|c|c|c|c|}
\hline \multicolumn{2}{|c|}{ Treatments } & \multicolumn{2}{|c|}{$\begin{array}{l}\text { Root length. } \\
\text { (cm) }\end{array}$} & \multicolumn{2}{|c|}{$\begin{array}{l}\text { Plant height. } \\
\text { (cm) }\end{array}$} & \multicolumn{2}{|c|}{$\begin{array}{l}\text { Leaves } \\
\text { Number. }\end{array}$} & \multicolumn{2}{|c|}{ Leaf area. $\left(\mathrm{cm}^{2}\right)$} \\
\hline $\begin{array}{c}\text { Heavy } \\
\text { metals } \\
(\mathrm{ppm})\end{array}$ & $\begin{array}{c}\text { Inducers } \\
\text { (ppm) }\end{array}$ & $\begin{array}{c}\text { Season } \\
1\end{array}$ & $\begin{array}{c}\text { Season } \\
\text { II }\end{array}$ & $\begin{array}{c}\text { Season } \\
I\end{array}$ & $\begin{array}{c}\text { Season } \\
\text { II }\end{array}$ & $\begin{array}{c}\text { Season } \\
I\end{array}$ & $\begin{array}{c}\text { Season } \\
\text { II }\end{array}$ & $\begin{array}{c}\text { Season } \\
1\end{array}$ & $\begin{array}{c}\text { Season } \\
\text { II }\end{array}$ \\
\hline 0 & - & 16.33 & 21.00 & 52.50 & 53.50 & 10.83 & 11.17 & 370.63 & 262.50 \\
\hline $\mathrm{Pb} 1000$ & - & 15.67 & 15.33 & 50.50 & 51.00 & 9.67 & 9.17 & 307.39 & 206.16 \\
\hline Pb2000 & - & 13.83 & 13.67 & 47.17 & 44.17 & 9.00 & 8.83 & 158.68 & 140.60 \\
\hline Cd100 & - & 15.17 & 14.17 & 48.83 & 48.33 & 9.17 & 9.00 & 217.32 & 166.00 \\
\hline Cd300 & - & 11.50 & 11.50 & 41.33 & 41.17 & 8.17 & 8.33 & 122.18 & 63.17 \\
\hline \multicolumn{2}{|c|}{ LSD at $5 \%$} & 0.215 & 0.254 & 0.364 & 0.242 & ns & ns & 2.923 & 5.094 \\
\hline- & 0 & 14.00 & 14.10 & 46.90 & 45.70 & 9.10 & 9.00 & 209.34 & 141.56 \\
\hline- & Si & 14.40 & 14.80 & 48.10 & 47.90 & 9.40 & 9.20 & 221.43 & 169.12 \\
\hline - & Sw & 15.10 & 16.50 & 49.20 & 49.30 & 9.60 & 9.70 & 274.94 & 192.37 \\
\hline \multicolumn{2}{|c|}{ LSD at $5 \%$} & 0.124 & 0.117 & 0.248 & 0.179 & ns & ns & 0.583 & 0.634 \\
\hline \multirow{3}{*}{0} & 0 & 16.0 & 19.0 & 51.5 & 51.5 & 10.5 & 10.5 & 319.75 & 230.70 \\
\hline & Si & 16.5 & 20.0 & 52.0 & 52.5 & 11.0 & 10.5 & 327.36 & 249.78 \\
\hline & Sw & 16.5 & 24.0 & 54.0 & 56.5 & 11.0 & 12.5 & 464.77 & 307.03 \\
\hline \multirow{3}{*}{ Pb1000 } & 0 & 15.5 & 14.5 & 50.5 & 50.0 & 9.5 & 9.0 & 301.41 & 175.75 \\
\hline & Si & 15.5 & 14.5 & 50.5 & 51.5 & 9.5 & 9.0 & 305.36 & 218.18 \\
\hline & Sw & 16.0 & 17.0 & 50.5 & 51.5 & 10.0 & 9.5 & 315.40 & 224.54 \\
\hline \multirow{3}{*}{$\mathrm{Pb} 2000$} & 0 & 13.0 & 13.5 & 46.0 & 44.0 & 9.0 & 8.5 & 145.56 & 116.61 \\
\hline & Si & 14.0 & 13.5 & 47.5 & 44.0 & 9.0 & 9.0 & $\mid 156.48$ & 147.73 \\
\hline & Sw & 14.5 & 14.0 & 48.0 & 44.5 & 9.0 & 9.0 & 174.00 & 157.45 \\
\hline \multirow{3}{*}{ Cd100 } & 0 & 15.0 & 14.0 & 48.0 & 46.0 & 9.0 & 9.0 & 176.50 & 161.67 \\
\hline & Si & 15.0 & 14.0 & 48.0 & 49.0 & 9.0 & 9.0 & 189.67 & 163.73 \\
\hline & Sw & 15.5 & 14.5 & 50.5 & 50.0 & 9.5 & 9.0 & 285.79 & 172.59 \\
\hline $\mathrm{Cd} 300$ & 0 & 10.5 & 9.5 & 38.5 & 37.0 & 7.5 & 8.0 & 103.50 & 23.06 \\
\hline
\end{tabular}

compared with $\mathrm{Pb}$ concentrations and $\mathrm{Cd}$, which caused a marked increase in this respect by about, $88 \%$ at the first season compared to its control. 
Physiological studies on the heavy metals resistance mechanisms in tomato

\begin{tabular}{|c|c|c|c|c|c|c|c|c|c|}
\hline & $\mathrm{Si}$ & 11.0 & 12.0 & 42.5 & 42.5 & 8.5 & 8.5 & 128.28 & 66.20 \\
\cline { 2 - 9 } & $\mathrm{Sw}$ & 13.0 & 13.0 & 43.0 & 44.0 & 8.5 & 8.5 & 134.75 & 100.25 \\
\hline LSD at 5\% & 0.087 & 0.069 & 0.078 & 0.090 & $\mathrm{~ns}$ & $\mathrm{~ns}$ & 0.036 & 0.031 \\
\hline
\end{tabular}

Table (3): Effect of heavy metals, nanoparticles and their interactions on dry weight of tomato plants at 60 days from transplanting during the growing seasons of 2018 and 2019.

\begin{tabular}{|c|c|c|c|c|c|c|c|c|c|}
\hline \multicolumn{2}{|c|}{ Treatments $\quad$ Characters } & \multicolumn{4}{|c|}{$\begin{array}{l}\text { Dry weight (g) } \\
\text { season I }\end{array}$} & \multicolumn{4}{|c|}{$\begin{array}{l}\text { Dry weight (g) } \\
\text { season II }\end{array}$} \\
\hline $\begin{array}{l}\text { Heavy metals } \\
\quad(p p m)\end{array}$ & $\begin{array}{c}\text { Inducers } \\
\text { (ppm) }\end{array}$ & Root & Stem & Leaf & Whole & Root & Stem & Leaf & Whole \\
\hline 0 & - & 2.10 & 1.76 & 4.69 & 8.56 & 2.23 & 1.66 & 4.40 & 8.29 \\
\hline Pb1000 & - & 1.47 & 1.28 & 3.64 & 6.40 & 1.30 & 1.58 & 3.55 & 6.43 \\
\hline $\mathrm{Pb} 2000$ & - & 0.59 & 1.09 & 2.41 & 4.09 & 0.58 & 0.94 & 2.09 & 3.61 \\
\hline Cd100 & - & 0.79 & 1.17 & 2.97 & 4.93 & 0.98 & 1.33 & 3.22 & 5.53 \\
\hline $\mathrm{Cd} 300$ & - & 0.52 & 0.90 & 1.88 & 3.30 & 0.39 & 0.71 & 0.94 & 2.05 \\
\hline \multicolumn{2}{|c|}{ LSD at $5 \%$} & 0.069 & 0.079 & 0.526 & 0.784 & 0.147 & 0.062 & 0.256 & 0.698 \\
\hline - & 0 & 0.94 & 1.16 & 2.82 & 4.92 & 0.87 & 1.20 & 2.48 & 4.55 \\
\hline- & Si & 1.07 & 1.20 & 3.08 & 5.35 & 0.98 & 1.22 & 2.86 & 5.07 \\
\hline - & Sw & 1.27 & 1.36 & 3.47 & 6.09 & 1.43 & 1.32 & 3.18 & 5.93 \\
\hline \multicolumn{2}{|c|}{ LSD at $5 \%$} & 0.129 & 0.040 & 0.258 & 0.427 & 0.085 & 0.016 & 0.248 & 0.403 \\
\hline \multirow{3}{*}{0} & 0 & 1.80 & 1.57 & 4.03 & 7.40 & 1.54 & 1.65 & 3.89 & 7.08 \\
\hline & Si & 2.03 & 1.60 & 4.58 & 8.21 & 1.74 & 1.66 & 4.50 & 7.90 \\
\hline & Sw & 2.47 & 2.12 & 5.47 & 10.06 & 3.41 & 1.67 & 4.80 & 9.88 \\
\hline \multirow{3}{*}{ Pb1000 } & 0 & 1.29 & 1.24 & 3.42 & 5.95 & 1.13 & 1.55 & 3.44 & 6.12 \\
\hline & $\mathrm{Si}$ & 1.45 & 1.24 & 3.67 & 6.36 & 1.29 & 1.59 & 3.52 & 6.40 \\
\hline & Sw & 1.68 & 1.37 & 3.84 & 6.89 & 1.48 & 1.61 & 3.68 & 6.77 \\
\hline \multirow{3}{*}{ Pb2000 } & 0 & 0.57 & 1.03 & 2.30 & 3.90 & 0.44 & 0.89 & 1.60 & 2.93 \\
\hline & Si & 0.58 & 1.12 & 2.32 & 4.02 & 0.49 & 0.93 & 2.18 & 3.60 \\
\hline & Sw & 0.61 & 1.13 & 2.62 & 4.36 & 0.82 & 1.01 & 2.48 & 4.31 \\
\hline \multirow{3}{*}{ Cd100 } & 0 & 0.62 & 1.14 & 2.82 & 4.58 & 0.89 & 1.27 & 3.08 & 5.24 \\
\hline & Si & 0.73 & 1.15 & 2.85 & 4.73 & 0.99 & 1.28 & 3.15 & 5.42 \\
\hline & Sw & 1.01 & 1.22 & 3.24 & 5.47 & 1.05 & 1.45 & 3.44 & 5.94 \\
\hline $\mathrm{Cd} 300$ & 0 & 0.43 & 0.84 & 1.52 & 2.79 & 0.37 & 0.65 & 0.37 & 1.39 \\
\hline
\end{tabular}


A.M. Abd El-All, et al.,

\begin{tabular}{|c|c|c|c|c|c|c|c|c|c|}
\hline & Si & 0.56 & 0.90 & 1.97 & 3.43 & 0.40 & 0.65 & 0.97 & 2.02 \\
\cline { 2 - 9 } & Sw & 0.57 & 0.95 & 2.16 & 3.68 & 0.41 & 0.84 & 1.48 & 2.73 \\
\hline \multicolumn{2}{|c|}{ LSD at 5\% } & 0.012 & 0.010 & 0.020 & 0.119 & 0.008 & 0.008 & 0.054 & 0.140 \\
\hline
\end{tabular}

Table (4): Effect of heavy metals, nanoparticles and their interactions on relative water content (RWC) and membrane integrity (MI) of tomato plants at 60 days from transplanting during the growing seasons of 2018 and 2019.

\begin{tabular}{|c|c|c|c|c|c|}
\hline \multicolumn{2}{|c|}{$\begin{array}{ll} & \text { Characters } \\
\end{array}$} & \multicolumn{2}{|c|}{ RWC (\%) } & \multicolumn{2}{|c|}{ MI (\%) } \\
\hline Heavy metals (ppm) & Inducers (ppm) & I season & II season & I season & II season \\
\hline 0 & - & 75.68 & 72.29 & 25.98 & 32.50 \\
\hline $\mathrm{Pb} 1000$ & - & 73.35 & 64.57 & 33.99 & 44.86 \\
\hline Pb2000 & - & 69.59 & 55.81 & 42.25 & 49.35 \\
\hline Cd100 & - & 72.39 & 61.36 & 38.99 & 48.03 \\
\hline Cd300 & - & 59.58 & 50.66 & 48.76 & 51.52 \\
\hline \multicolumn{2}{|c|}{ LSD at $5 \%$} & 0.671 & 2.702 & 0.660 & 1.649 \\
\hline- & 0 & 68.16 & 59.47 & 39.83 & 47.48 \\
\hline- & Si & 70.37 & 61.03 & 38.20 & 45.50 \\
\hline- & SWE & 71.82 & 62.32 & 35.96 & 42.78 \\
\hline \multicolumn{2}{|c|}{ LSD at $5 \%$} & 0.438 & 1.146 & 0.876 & 1.493 \\
\hline \multirow{3}{*}{0} & 0 & 74.45 & 70.09 & 29.15 & 38.58 \\
\hline & Si & 76.03 & 72.41 & 26.84 & 34.93 \\
\hline & SWE & 76.57 & 74.36 & 21.94 & 23.99 \\
\hline \multirow{3}{*}{ Pb1000 } & 0 & 72.94 & 62.48 & 35.64 & 47.37 \\
\hline & Si & 73.44 & 64.73 & 33.63 & 43.60 \\
\hline & SWE & 73.67 & 66.50 & 32.71 & 43.60 \\
\hline \multirow{3}{*}{ Pb2000 } & 0 & 68.60 & 54.66 & 43.76 & 49.56 \\
\hline & Si & 68.64 & 55.87 & 42.47 & 49.52 \\
\hline & Sw & 71.52 & 56.90 & 40.51 & 48.97 \\
\hline \multirow{3}{*}{ Cd100 } & 0 & 72.04 & 60.52 & 39.45 & 48.94 \\
\hline & Si & 72.34 & 61.75 & 39.30 & 47.76 \\
\hline & SWE & 72.80 & 61.82 & 38.22 & 47.40 \\
\hline
\end{tabular}


Physiological studies on the heavy metals resistance mechanisms in tomato ......

\begin{tabular}{|c|c|c|c|c|c|}
\hline \multirow{3}{*}{ Cd300 } & 0 & 52.77 & 49.58 & 51.14 & 52.95 \\
\hline & Si & 61.42 & 50.37 & 48.74 & 51.68 \\
\hline & SWE & 64.56 & 52.03 & 46.41 & 49.92 \\
\hline \multicolumn{2}{|c|}{ LSD at $5 \%$} & 0.284 & 0.875 & 0.077 & 0.933 \\
\hline
\end{tabular}

It was noticed from data presented in Table (4) that, relative water content was significantly increased with $\mathrm{Si}$ and SWE treatments on tomato plants as compared with the control which reached about $5 \%$ at the first season. Results in the second season are like to the first season. Meanwhile, Si and SWE extract caused a remarkable decrease in $\mathrm{MI}$, the highest decrease in tomato MI was recorded with SWE treatment by about $88 \%$ at the first season as compared with the untreated plants.

As for the effect of the interaction between $(\mathrm{Pb}$ and $\mathrm{Cd}$ ) and (SWE and $\mathrm{Si}$ ) were observed in Table (4) citied that, all levels of $\mathrm{Pb}$ and $\mathrm{Cd}$ caused a decrease in relative water content and increase $\mathrm{MI}$ in tomato leaves, seaweed and silicon caused a recovering to a deleterious effects of heavy metals when spraying on tomato plants, grown in contaminated soil with $\mathrm{Pb}$ and $\mathrm{Cd}$ when compared with the control plants. These results were confirmed in the second season.

Similar findings have been demonstrated by Sobkowiak (2016) who mentioned that, heavy metals or toxic ions reduced root hair and had a deleterious effect on the root-absorbing area and water uptake. Moreover, metals are able to decelerate short-distance water transfer both in symplast and apoplast, which reduce the movement of water into the vascular system and affect water content, which decreased in various organs. These results are in agreement with the flinging of Khan et al. (2020) demonstrated that, nano Si application reduced the oxidative stress caused by $\mathbf{C d}$ in leaves was indicated by the reduced production of hydrogen peroxide and electrolyte leakage.

\section{Photosynthetic pigments:}

The obtained results in Tables (5 a and $\mathrm{b})$ showed that, all levels of $\mathrm{Pb}$ and Cd decreased the concentrations of photosynthetic pigments (i. e. chlorophyll $a, b, a+b$ and carotenoids) in leaves of tomato plants, meanwhile the ratios of a / $b$ and $a+b /$ carotenoids showed an uncorrupted results at the levels of $\mathrm{Pb}$ an Cd.

Data illustrated that, foliar application of silicon and seaweed, had a significant increase in leaf chlorophylls and carotenoids concentrations of tomato plants. Marked effects were noticed in the ratios of chl. a / b and chl. a + b / carotenoids.

The interaction data showed that, all levels of different heavy metals caused a decrease in tomato chl. a, b, a + b and carotenoids, but silicon and seaweed caused a recovering to this decrease which caused by heavy metals when spraying on tomato plants, and grown in contaminated soil with heavy metals when compared with the untreated plants. The same results were found in the second season. These results are according to the findings by Saad-Allah et al. (2016) who found that, Cd stress caused a significant reduction in, photosynthetic pigments, however, presoaking in seaweed extract enhanced the photosynthetic pigments in case of control plants. Additionally, the interactive combination of cadmium stress and algal extract showed a 
A.M. Abd El-All, et al.,

significant amendment of cadmium stress on photosynthetic pigments. Suriyaprabha et al. (2012) reported that, absorption of nano silicon dioxide

significant increase in organic compounds such as proteins, chlorophyell and phenols and plant dry weight.

Table (5 a): Effect of heavy metals, nanoparticles and their interactions on photosynthetic pigments in leaves of tomato plants at 60 days from transplanting during the growing season of 2018 .

\begin{tabular}{|c|c|c|c|c|c|c|c|}
\hline \multicolumn{2}{|c|}{$\begin{array}{ll}\text { Treatments } & \text { Characters } \\
\end{array}$} & \multirow{2}{*}{$\begin{array}{l}\text { Chl.a } \\
\text { (mg/g } \\
\text { dwt) }\end{array}$} & \multirow{2}{*}{$\begin{array}{l}\text { Chl.b } \\
(\mathrm{mg} / \mathrm{g} \\
\mathrm{dwt})\end{array}$} & \multirow{2}{*}{$\begin{array}{l}\text { Caro. } \\
(\mathrm{mg} / \mathrm{g} \\
\mathrm{dwt})\end{array}$} & \multirow{2}{*}{$\begin{array}{c}\text { Total } \\
\text { Chl } \\
\text { (mg/g } \\
\text { dwt) }\end{array}$} & \multirow{2}{*}{ Chla/b } & \multirow{2}{*}{$\begin{array}{c}\text { T.Chl/Ca } \\
\text { r. }\end{array}$} \\
\hline $\begin{array}{l}\text { Heavy metals } \\
\quad(p p m)\end{array}$ & $\begin{array}{l}\text { Inducers } \\
\text { (ppm) }\end{array}$ & & & & & & \\
\hline & & \multicolumn{6}{|c|}{ Season 2018} \\
\hline 0 & - & 8.18 & 5.00 & 6.43 & 13.18 & 1.71 & 2.15 \\
\hline Pb1000 & - & 4.65 & 2.31 & 3.88 & 6.96 & 2.08 & 1.79 \\
\hline Pb2000 & - & 3.23 & 1.59 & 2.07 & 4.82 & 2.02 & 2.33 \\
\hline Cd100 & - & 4.13 & 1.74 & 2.98 & 5.87 & 2.37 & 2.00 \\
\hline $\mathrm{Cd} 300$ & - & 2.48 & 1.49 & 1.64 & 3.97 & 1.66 & 2.57 \\
\hline \multicolumn{2}{|l|}{ LSD at $5 \%$} & 0.281 & 0.186 & 0.012 & 0.148 & 0.393 & 0.002 \\
\hline- & 0 & 4.23 & 2.03 & 2.68 & 6.26 & 2.05 & 2.47 \\
\hline- & Si & 4.55 & 2.35 & 3.37 & 6.90 & 2.02 & 2.07 \\
\hline- & SWE & 4.82 & 2.91 & 4.15 & 7.73 & 1.84 & 1.96 \\
\hline \multicolumn{2}{|l|}{ LSD at $5 \%$} & 0.211 & 0.023 & 1.243 & 0.932 & 0.191 & 0.017 \\
\hline \multirow{3}{*}{0} & 0 & 7.58 & 3.53 & 4.35 & 11.11 & 2.15 & 2.55 \\
\hline & Si & 8.18 & 4.95 & 6.00 & 13.13 & 1.65 & 2.19 \\
\hline & SWE & 8.78 & 6.53 & 8.93 & 15.31 & 1.34 & 1.71 \\
\hline \multirow{3}{*}{ Pb1000 } & 0 & 4.35 & 1.95 & 3.53 & 6.30 & 2.23 & 1.78 \\
\hline & Si & 4.73 & 1.98 & 3.98 & 6.71 & 2.39 & 1.69 \\
\hline & SWE & 4.88 & 3.00 & 4.13 & 7.88 & 1.63 & 1.91 \\
\hline \multirow{3}{*}{ Pb2000 } & 0 & 2.85 & 1.58 & 1.98 & 4.43 & 1.80 & 2.24 \\
\hline & Si & 3.38 & 1.60 & 1.99 & 4.98 & 2.11 & 2.50 \\
\hline & SWE & 3.45 & 1.60 & 2.25 & 5.05 & 2.16 & 2.24 \\
\hline \multirow{3}{*}{ Cd100 } & 0 & 4.05 & 1.65 & 2.48 & 5.70 & 2.45 & 2.30 \\
\hline & Si & 4.05 & 1.75 & 3.00 & 5.80 & 2.31 & 1.93 \\
\hline & SWE & 4.28 & 1.83 & 3.45 & 6.11 & 2.34 & 1.77 \\
\hline
\end{tabular}


Physiological studies on the heavy metals resistance mechanisms in tomato .......

\begin{tabular}{|l|c|c|c|c|c|c|c|}
\hline \multirow{3}{*}{$\mathrm{Cd} 300$} & 0 & 2.33 & 1.43 & 1.08 & 3.76 & 1.63 & 3.48 \\
\cline { 2 - 7 } & $\mathrm{Si}$ & 2.40 & 1.47 & 1.88 & 3.87 & 1.63 & 2.06 \\
\cline { 2 - 7 } & $\mathrm{SWE}$ & 2.70 & 1.58 & 1.97 & 4.28 & 1.71 & 2.17 \\
\hline LSD at 5\% & & 0.174 & 0.014 & 0.011 & 0.095 & 0.115 & 0.002 \\
\hline
\end{tabular}

Table $(5 \mathrm{~b})$ : Effect of heavy metals, nanoparticles and their interactions on photosynthetic pigments in leaves of tomato plants at 60 days from transplanting during the growing season of 2019.

\begin{tabular}{|c|c|c|c|c|c|c|c|}
\hline & & & & Seas & 2019 & & \\
\hline 0 & - & 5.90 & 2.18 & 3.98 & 8.08 & 2.71 & 2.03 \\
\hline Pb1000 & - & 4.53 & 1.82 & 3.05 & 6.35 & 2.48 & 2.07 \\
\hline Pb2000 & - & 2.30 & 1.18 & 2.15 & 3.48 & 1.94 & 1.62 \\
\hline Cd100 & - & 3.25 & 1.35 & 2.38 & 4.60 & 2.41 & 1.93 \\
\hline $\mathrm{Cd} 300$ & - & 1.45 & 1.08 & 1.45 & 2.53 & 1.34 & 1.82 \\
\hline LSD at $5^{\circ}$ & & 0.135 & 0.325 & 0.821 & 1.524 & 0.045 & 0.027 \\
\hline- & 0 & 3.11 & 1.46 & 2.42 & 4.56 & 1.99 & 1.87 \\
\hline- & Si & 3.32 & 1.50 & 2.55 & 4.81 & 2.10 & 1.88 \\
\hline- & SWE & 4.04 & 1.61 & 2.84 & 5.65 & 2.43 & 1.95 \\
\hline LSD at $5^{\circ}$ & & 0.072 & 0.198 & 0.433 & 0.803 & 0.036 & 0.018 \\
\hline & 0 & 5.63 & 2.03 & 3.75 & 7.66 & 2.77 & 2.04 \\
\hline 0 & Si & 5.78 & 2.18 & 3.90 & 7.96 & 2.65 & 2.04 \\
\hline & SWE & 6.30 & 2.33 & 4.28 & 8.63 & 2.70 & 2.02 \\
\hline & 0 & 4.05 & 1.73 & 2.93 & 5.78 & 2.34 & 1.97 \\
\hline Pb1000 & $\mathbf{S i}$ & 4.13 & 1.75 & 3.08 & 5.88 & 2.36 & 1.91 \\
\hline & SWE & 5.40 & 1.98 & 3.15 & 7.38 & 2.73 & 2.34 \\
\hline & 0 & 2.10 & 1.13 & 2.12 & 3.23 & 1.86 & 1.52 \\
\hline Pb2000 & Si & 2.10 & 1.13 & 2.15 & 3.23 & 1.86 & 1.50 \\
\hline & SWE & 2.70 & 1.28 & 2.18 & 3.98 & 2.11 & 1.83 \\
\hline & 0 & 2.70 & 1.35 & 2.25 & 4.05 & 2.00 & 1.80 \\
\hline Cd100 & Si & 3.23 & 1.35 & 2.40 & 4.58 & 2.39 & 1.91 \\
\hline & SWE & 3.83 & 1.35 & 2.48 & 5.18 & 2.84 & 2.09 \\
\hline $\mathrm{Cd} 300$ & 0 & 1.05 & 1.05 & 1.05 & 2.10 & 1.00 & 2.00 \\
\hline
\end{tabular}


A.M. Abd El-All, et al.,

\begin{tabular}{|l|c|c|c|c|c|c|c|}
\hline & Si & 1.35 & 1.07 & 1.20 & 2.42 & 1.26 & 2.02 \\
\cline { 2 - 8 } & SWE & 1.95 & 1.11 & 2.10 & 3.06 & 1.76 & 1.46 \\
\hline LSD at 5\% & & 0.011 & 0.023 & 0.031 & 0.159 & 0.009 & 0.008 \\
\hline
\end{tabular}

\section{Antioxidant Enzymes Activity:-}

Results in Table (6) showed that, all concentrations of $\mathrm{Pb}$ and $\mathrm{Cd}$ significantly increased the peroxidase enzyme activity on leaves of tomato plants which recorded by $\mathbf{7 0} \%$ with $300 \mathrm{ppm} \mathrm{Cd}$ in the first season when compared with the control. Meanwhile a great increase in the activity of phenoloxidase enzyme, this increase was more pronounced at level of Cd 300 which recorded by $83 \%$ compared with the control in the first season. The same results in the second season like the first one.

Table (6): Effect of heavy metals, nanoparticles and their interactions on antioxidant enzymes activities (OD) in leaves of tomato plants at 60 days from transplanting during the growing seasons of 2018 and 2019.

\begin{tabular}{|c|c|c|c|c|c|}
\hline \multicolumn{2}{|c|}{$\begin{array}{ll}\text { Treatments } & \text { Characters } \\
\end{array}$} & \multicolumn{2}{|c|}{$\begin{array}{c}\text { Peroxidase } \\
\text { (OD after } 2 \mathrm{mint} / \mathrm{g} \mathrm{fw} \text { ) }\end{array}$} & \multicolumn{2}{|c|}{$\begin{array}{c}\text { Phenoloxidase } \\
\text { (OD after } 45 \mathrm{mint} / \mathrm{g} \mathrm{fw} \text { ) }\end{array}$} \\
\hline $\begin{array}{l}\text { Heavy metals } \\
\quad(p p m)\end{array}$ & Inducers (ppm) & season I & season II & season I & season II \\
\hline 0 & - & 0.430 & 0.374 & 1.111 & 1.000 \\
\hline Pb1000 & - & 0.555 & 0.426 & 1.296 & 1.370 \\
\hline $\mathrm{Pb} 2000$ & - & 0.651 & 0.496 & 1.704 & 2.222 \\
\hline Cd100 & - & 0.618 & 0.471 & 1.555 & 2.000 \\
\hline $\mathrm{Cd} 300$ & - & 0.729 & 0.541 & 2.037 & 2.222 \\
\hline \multicolumn{2}{|c|}{ LSD at $5 \%$} & 0.030 & 0.023 & 0.057 & 0.041 \\
\hline- & 0 & 0.635 & 0.476 & 1.644 & 1.866 \\
\hline- & $\mathbf{S i}$ & 0.589 & 0.462 & 1.578 & 1.766 \\
\hline- & SWE & 0.566 & 0.447 & 1.400 & 1.655 \\
\hline \multicolumn{2}{|c|}{ LSD at $5 \%$} & 0.021 & 0.013 & 0.611 & 0.038 \\
\hline \multirow{3}{*}{0} & 0 & 0.478 & 0.389 & 1.222 & 1.222 \\
\hline & $\mathrm{Si}$ & 0.411 & 0.367 & 1.222 & 0.944 \\
\hline & SWE & 0.400 & 0.367 & 0.889 & 0.833 \\
\hline \multirow{3}{*}{ Pb1000 } & 0 & 0.578 & 0.433 & 1.333 & 1.444 \\
\hline & Si & 0.544 & 0.433 & 1.333 & 1.333 \\
\hline & SWE & 0.544 & 0.411 & 1.222 & 1.333 \\
\hline \multirow{3}{*}{ Pb2000 } & 0 & 0.655 & 0.500 & 1.778 & 2.222 \\
\hline & Si & 0.655 & 0.500 & 1.667 & 2.222 \\
\hline & SWE & 0.644 & 0.489 & 1.667 & 2.222 \\
\hline \multirow{2}{*}{ Cd100 } & 0 & 0.644 & 0.489 & 1.667 & 2.222 \\
\hline & Si & 0.622 & 0.467 & 1.555 & 2.111 \\
\hline
\end{tabular}


Physiological studies on the heavy metals resistance mechanisms in tomato .......

\begin{tabular}{|c|c|c|c|c|c|}
\hline & SWE & 0.589 & 0.456 & 1.444 & 1.667 \\
\hline \multirow{3}{*}{ Cd300 } & 0 & 0.822 & 0.567 & 2.222 & 2.222 \\
\cline { 2 - 6 } & Si & 0.711 & 0.544 & 2.111 & 2.222 \\
\cline { 2 - 6 } & SWE & 0.655 & 0.511 & 1.778 & 2.222 \\
\hline \multicolumn{2}{|c|}{ LSD at 5\% } & 0.015 & 0.011 & 0.134 & 0.029 \\
\hline
\end{tabular}

Data recorded in Table (6) cleared that, SWE and Si caused a remarkable decrease in the peroxidase enzyme activity in leaves of tomato plants. Also, it can be observed from the same table peroxidase enzyme activity in leaves was decreased under the same treatment with nanoparticles which recorded by $11 \%$ in peroxidase enzyme activity and $15 \%$ in phenoloxidase enzyme activity compared with the control plants. The second season is similar to the first one.

These results are in accordance to those recorded by Saeed et al. (2014) who mentioned that, application cadmium to tomato plants resulted in increasing peroxidatase activity by $72 \%$ and ascorbic acid oxidase by $12 \%$ compared to the control plants. The obtained results confirmed by those recorded by Tripathi et al. (2016) who said that, under $\mathrm{Cd}$ stress and $\mathrm{Si}$ application enhances the activities of antioxidant enzymes and reduces the oxidative stress by decreasing the malondialdehyde (MDA). Silicon also reduces $\mathrm{Cd}$ toxicity by structural alterations in plants and by regulating gene expression.

As for the effect of the interaction between (Pb and $\mathrm{Cd}$ ) and (SWE and $\mathrm{Si}$ ) were observed in Table (6) citied that, all levels of $\mathrm{Pb}$ and $\mathrm{Cd}$ caused an increased in oxidase enzyme activity and peroxidase enzyme activity in tomato leaves, but seaweed and silver caused a recovering to a deleterious effects of heavy metals when spraying on tomato plants, and which grown in contaminated soil with $\mathrm{Pb}$ and $\mathrm{Cd}$ when compared with the control plants. These results were confirmed in the second season. Similar findings have been demonstrated by Shuyi et al. (2013) showed that, spraying silicon could enhance activities of SOD and POD. Zargar et al. (2019) reported that, nano Si treatment had a stimulation of enzymatic and non-enzymatic antioxidants enzymes in rice shoots which decreased $\mathrm{Cd}$ accumulation. Moreover, Si supply reduces lipid peroxidation (LPO) intensity whereas increases enzymatic (superoxide dismutase, SOD, APX and glutathione reductase) and non-enzymatic (ascorbate and glutathiome) antioxidants activities in cucumber (Shi et al. 2005, Maksimovic et al. 2012 and Khan et al. 2020).

\section{Accumulation of heavy metals by watercress plants grown under contaminated soil by $\mathbf{P b}$ and $\mathrm{Cd}$ :}

The obtained results in Table (7) \& Fig 1 cleared that, the accumulation of lead and cadmium by watercress plants increased with increasing the concentration of $\mathrm{Pb}$ from 1000 to 2000ppm and Cd from 100 to $300 \mathrm{ppm}$. The residual concentration of lead and cadmium in polluted soil after the harvest plants increased with increasing the level of $\mathrm{Pb}$ and $\mathrm{Cd}$. The highest accumulation in whole plants of watercress was recorded at the $\mathrm{Pb}$ level of 2000ppm and Cd level of $300 \mathrm{ppm}$. As well as the highest level of the soil residual of $\mathrm{Pb}$ and $\mathrm{Cd}$ were observed in the highest same levels of $\mathrm{Pb}$ and $\mathrm{Cd}$ after harvest crop, if compared with their control plants. The same trend was obtained in the second season. These results are in 
agreement with those obtained by Liu et al. (2009) on wheat plants, Fazal et al. (2010) on maize plants and Yongsheng et al. (2011) showed that, the ability of $\mathrm{Pb}$ accumulation of tea plant, having a positive correlation with $\mathrm{Pb}(800,1100$, 1400, 1700 and $2100 \mathrm{mg} / \mathrm{kg}$ soil) treatment concentrations, was in the

Table (7): Accumulation of heavy metals by Watercress and Tomato plants grown under contaminated soil by $\mathrm{Pb}$ and $\mathrm{Cd}$ during the growing seasons of 2018 and 2019.

\begin{tabular}{|c|c|c|c|c|c|}
\hline \multirow{2}{*}{ Characters } & \multicolumn{3}{|c|}{ Tomato $\mathrm{mg} / \mathrm{g}^{-1}$ dry wt. } & \multirow{2}{*}{$\begin{array}{l}\text { Watercress whole } \\
\text { / pot }\left(\mathrm{mg} / \mathrm{g}^{-1} \text { dry wt. }\right. \\
\text { heavy metals) }\end{array}$} & \multirow{2}{*}{$\begin{array}{c}\text { Soil } \\
\text { Residual (mg / pot } \\
\text { heavy metals) }\end{array}$} \\
\hline & Root & Shoot & Fruits & & \\
\hline & \multicolumn{5}{|c|}{ Season I } \\
\hline Control & 0 & 0 & 0.00 & 0.48 & 0.00 \\
\hline Pb1000 & 84 & 55 & 0.01 & 5448 & 2408 \\
\hline Pb1000+si & 61 & 40 & 0.00 & 57398 & 2150 \\
\hline Pb1000+SWE & 47 & 31 & 0.00 & 5968 & 1949 \\
\hline $\mathrm{Pb} 2000$ & 255 & 168 & 0.02 & 4974 & 10596 \\
\hline Pb2000+si & 175 & 116 & 0.00 & 6721 & 8982 \\
\hline Pb2000+SWE & 147 & 97 & 0.00 & 8162 & 7571 \\
\hline Cd100 & 10 & 7 & 0.03 & 554 & 226 \\
\hline Cd100+si & 8 & 5 & 0.00 & 568 & 219 \\
\hline Cd100+SWE & 6 & 4 & 0.00 & 582 & 206 \\
\hline Cd300 & 48 & 31 & 0.05 & 788 & 1532 \\
\hline Cd300+si & 33 & 22 & 0.00 & 876 & 1467 \\
\hline \multirow[t]{2}{*}{ Cd300+SWE } & 28 & 18 & 0.00 & 1141 & 1210 \\
\hline & \multicolumn{5}{|c|}{ Season II } \\
\hline Control & 0 & 0 & 0.00 & 0.32 & 0.00 \\
\hline Pb1000 & 94 & 62 & 0.02 & 5172 & 2668 \\
\hline Pb1000+si & 68 & 45 & 0.00 & 5494 & 2384 \\
\hline Pb1000+SWE & 53 & 35 & 0.00 & 5747 & 2161 \\
\hline Pb2000 & 285 & 188 & 0.02 & 3772 & 11748 \\
\hline $\mathrm{Pb2000+si}$ & 196 & 129 & 0.00 & 5705 & 9964 \\
\hline Pb2000+SWE & 164 & 108 & 0.00 & 7308 & 8399 \\
\hline Cd100 & 12 & 8 & 0.03 & 528 & 251 \\
\hline
\end{tabular}

sequence of root>stem >shoot. Root was the main part of tea plant to fix $\mathrm{Pb}$. Yilmaz and Parlak (2011) on Groenlandia densa plants who found that, $\mathrm{Cd}$ concentrations in plants increased with increasing $\mathbf{C d}$ (0$20 \mathrm{mgL}^{-1}$ cadmium nitrate) supply levels. 
Physiological studies on the heavy metals resistance mechanisms in tomato .......

\begin{tabular}{|l|c|c|c|c|c|}
\hline Cd100+si & 9 & 7 & 0.00 & 543 & 242 \\
\hline Cd100+SWE & 7 & 4 & 0.00 & 559 & 228 \\
\hline & & & & & \\
\hline Cd300 & 53 & 35 & 0.04 & 613 & 1697 \\
\hline Cd300+si & 37 & 24 & 0.00 & 710 & 1627 \\
\hline Cd300+SWE & 31 & 20 & 0.00 & 1004 & 1342 \\
\hline
\end{tabular}
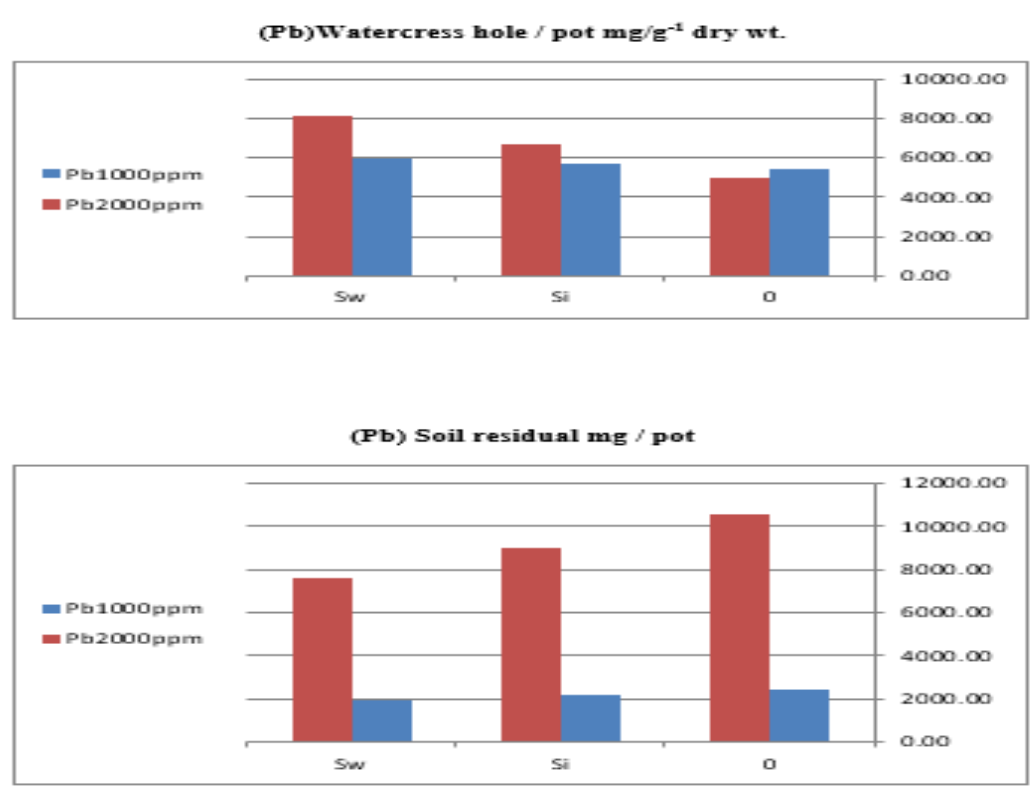

Fig 1: Effect of heavy metals, nanoparticles and on accumulation of $\mathrm{Pb}$ in Eruca plants and soil during the growing season of 2019.

Data in the Table (7) showed that, the ability of $\mathrm{Pb}$ and $\mathrm{Cd}$ accumulation in watercress plants, having a positive effect with seaweed and silicon treatments, as compared with the untreated plants. Meanwhile, the residual concentration of $\mathrm{Pb}$ and $\mathrm{Cd}$ in soil after the harvest crop decreased with applications of SWE and Si. The higher value of accumulation of $\mathrm{Pb}$ and $\mathrm{Cd}$ was observed at seaweed treatment. Similar findings have been demonstrated by Shuyi et al. (2013) and Dong et al. (2019) who found that, Pennisetum glaucum and Pennisetum glaucum - P. purpureum were used to explore the impacts of $\mathrm{Si}$ application on alleviating Cd toxicity and its possible mechanism. Treatments consist of four levels of $\mathrm{Cd}(0,10,50$, and $100 \mathrm{mg} \cdot \mathrm{kg}-1$ ) with or without $2.0 \mathrm{mM} \mathrm{Si}$ amendments. Under Cd stress, $\mathrm{Si}$ application significantly increased $\mathrm{Si}$ content and reduced $\mathrm{Cd}$ content.

Accumulation of heavy metals by tomato plants grown under contaminated soil by $\mathrm{Pb}$ and $\mathrm{Cd}$ :

The results in Table (7) cited that, the concentrations of lead and cadmium in root and shoot tomato plants increased with increasing the concentration levels of $\mathrm{Pb}$ and $\mathrm{Cd}$. The highest concentration of heavy metals in tomato plants was recorded at the $\mathrm{Pb}$ level of 2000ppm and 
Cd level of 300ppm. The concentrations of $\mathrm{Pb}$ and $\mathrm{Cd}$ in tomato roots were $\mathrm{a}$ higher than the concentrations in tomato shoots, but in tomato fruits were had a very low concentrate, if compared with their control plants. The same results were recorded in the second season. These results are in agreement with those obtained Kisa (2018) who pointed that, accumulation of metal ions in plants causes the formation of free radicals and stimulates the antioxidative defense systems.

Data in the Table (7) observed that, the silicon and seaweed foliar leaf applications caused a decrease in heavy metal concentration in roots and shoots of tomato plants which grown in polluted soil. The seaweed extract recorded a higher decrease in heavy metals content in tomato tissues, as compared with the untreated plants. The results in season one and two are the same. Our results are accordance with the findings by Goyal et al. (2017) who fond that, silica application can reduced toxic effects of heavy metal on $V$. radiata and improved its quality in terms of sugar and protein content.

Lead may enter the roots through several pathways, and a particular pathway is through ionic channels. Although, lead uptake is a non-selective phenomenon, it nonetheless depends on the functioning of an $\mathrm{H}^{+}$/ATPase pump to maintain a strong negative membrane potential in rhizoderm cells (Hirsch et al. 1998). Several authors have demonstrated that $\mathrm{Ca}^{2+}$-permeable channels are the main pathway by which lead enters roots (Wang et al. 2007).

\section{Yield and its components:}

Data presented in Tables (8a and $8 b$ ) showed that, fruit length, fruit around, fruit volume, fruits weight per plant, fruit number per plant and vitamin $C$ in tomato fruits were decreased at all treatments of
$\mathrm{Pb}$ and $\mathrm{Cd}$. The highest value of reduction was obtained at level of $\mathbf{C d} \mathbf{3 0 0}$ by about 52, 39, 63, 78 and $53 \%$, respectively. The same results were obtained in the second season.

From The obtained results in Tables ( $8 \mathrm{a}$ and $8 \mathrm{~b}$ ) it can be said that, SI and SW levels significantly increased the fruit length, fruit around, fruit volume, fruits weight per plant, fruit number per plant and vitamin $C$ in tomato fruit similar to those obtained in the first one.

The interactions between heavy metals and nanoparticles were illustrated in Tables (8a and $8 \mathrm{~b}$ ) and found that, all levels of $\mathrm{Pb}$ and $\mathrm{Cd}$ caused a decrease in tomato fruit length, fruit around, fruit volume, fruits weight per plant, fruit number per plant and vitamin $C$ in tomato fruits, but silicon and seaweed caused a recovering to deleterious effects which caused by heavy metals when treated plants of tomato, when compared with the untreated plants. The same result was observed in the $2^{\text {nd }}$ season.

The treatment of SW recorded a higher increase in these measures by about $12,8,15$ and $20 \%$. The results in the second season

Residual heavy metals (Cd, Co, Ni and $\mathrm{Pb}$ ) were examined in the harvested fruits. The obtained results were compared with those of tomato fruits cultivated in non-contaminated soil. Results demonstrate that soil contamination with heavy metals have a negative effect on tomato fruits characteristics. These results are in agreement with those recorded by Patel et al. (2020) observed that, papaya plants were sprayed with different concentrations of silicon (potassium silicate and ortho silicic acid at 0.2 and $0.4 \%$ ) and seaweed extract ( 2 and $4 \%$ ) either alone or in combinations at 3, 4, 5 and 6 months after planting. Application of potassium silicate at $0.4 \%$ + seaweed extract at $4 \%$ proved most effective in 
reducing physiological loss in weight and increasing shelf life with improved fruit firmness in papaya cv. Red Lady. Sensory parameters i.e. color, texture, flavor, taste, general appearance and overall acceptability were significantly better under foliar application of ortho silicic acid $0.2 \%$ + seaweed extract $2 \%$.

It can be observed that, all lead and cadmium treatments inhibited plant growth, water relations and chemical constituents in tomato plants. On the other hand the treated with heavy metals $(\mathrm{Pb}$ and $\mathrm{Cd})$ levels increased their concentration in roots and shoots of tomato led to negative effect on yield and its components. As well as plant pigments, TSS and minerals were decreased under contaminate conditions, thus the plant growth as well as the yield decreased. Similar results were obtained by Hashem et al. (2017) who mentioned that, on tomato plants. El-Gamal and Hammad (2003) too reported that, lead (250 and $500 \mathrm{mg} / \mathrm{l}$ ) and cadmium (100 and $200 \mathrm{mg} / \mathrm{l}$ ) negatively affected on fruit yield and fruit quality of tomato plants. Increase in heavy metal $\mathbf{~} \mathrm{Ni}, \mathrm{Cd}, \mathrm{Cu}, \mathrm{Cr}$, $\mathrm{Pb}$ and $\mathrm{Zn}$ ) concentration in foliage of plants grown in sewage sludge-amended soil caused unfavorable changes in physiological and biochemical characteristics of plants leading to reductions in yield (Singh and Agrawal, 2007).

Table (8a): Effect of heavy metals, nanoparticles and their interactions on yield and its components of tomato plants during the growing season of 2018.

\begin{tabular}{|c|c|c|c|c|c|c|c|}
\hline \multicolumn{2}{|l|}{ Treatments } & $\begin{array}{l}\text { Fruit } \\
\text { length } \\
\text { (cm) }\end{array}$ & $\begin{array}{l}\text { Fruit } \\
\text { around } \\
(\mathrm{cm})\end{array}$ & $\begin{array}{c}\begin{array}{c}\text { Fruit } \\
\text { volume } \\
\left(\mathrm{cm}^{3}\right)\end{array}\end{array}$ & $\begin{array}{c}\text { Fruits } \\
\text { weight / } \\
\text { plant } \\
\text { (gm) }\end{array}$ & $\begin{array}{c}\text { Fruit } \\
\text { N. / } \\
\text { plant }\end{array}$ & $\begin{array}{c}\text { Vitamin C. } \\
\text { (mg/100g } \\
\text { f.wt. } \\
\text { fruits) }\end{array}$ \\
\hline $\begin{array}{l}\text { Heavy metals } \\
(p p m)\end{array}$ & $\begin{array}{l}\text { Inducers } \\
\text { (ppm) }\end{array}$ & \multicolumn{6}{|c|}{ season I } \\
\hline 0 & - & 4.55 & 3.94 & 12.37 & 430.82 & 14.99 & 11.98 \\
\hline Pb1000 & - & 4.22 & 3.56 & 10.17 & 340.84 & 12.94 & 11.06 \\
\hline Pb2000 & - & 3.03 & 2.96 & 7.14 & 227.93 & 10.35 & 9.69 \\
\hline Cd100 & - & 3.72 & 3.16 & 8.06 & 273.53 & 11.75 & 10.24 \\
\hline Cd300 & - & 2.18 & 2.39 & 4.63 & 95.41 & 7.08 & 5.37 \\
\hline \multicolumn{2}{|c|}{ LSD at $5 \%$} & 0.640 & 0.186 & 0.853 & 42.301 & 1.299 & 0.052 \\
\hline- & 0 & 3.32 & 3.10 & 7.95 & 242.98 & 10.42 & 8.98 \\
\hline- & $\mathbf{S i}$ & 3.57 & 3.16 & 8.25 & 263.46 & 11.33 & 9.36 \\
\hline- & Sw & 3.73 & 3.35 & 9.21 & 314.69 & 12.51 & 10.66 \\
\hline \multicolumn{2}{|c|}{ LSD at $5 \%$} & 0.148 & 0.056 & 0.278 & 18.998 & 0.844 & 0.034 \\
\hline \multirow{3}{*}{0} & 0 & 4.38 & 3.82 & 11.97 & 379.11 & 13.83 & 11.15 \\
\hline & $\mathbf{S i}$ & 4.58 & 4.00 & 12.57 & 417.76 & 14.35 & 11.77 \\
\hline & Sw & 4.70 & 4.00 & 12.57 & 495.60 & 16.80 & 13.02 \\
\hline \multirow{3}{*}{ Pb1000 } & 0 & 4.10 & 3.50 & 9.63 & 333.36 & 12.10 & 11.37 \\
\hline & $\mathbf{S i}$ & 4.25 & 3.50 & 9.63 & 344.49 & 13.08 & 11.31 \\
\hline & Sw & 4.30 & 3.67 & 11.26 & 344.67 & 13.63 & 10.49 \\
\hline \multirow{3}{*}{ Pb2000 } & 0 & 2.98 & 2.93 & 6.95 & 202.66 & 9.82 & 8.94 \\
\hline & Si & 3.00 & 2.96 & 7.19 & 225.06 & 10.23 & 9.63 \\
\hline & Sw & 3.12 & 3.00 & 7.28 & 256.07 & 10.99 & 10.50 \\
\hline Cd100 & 0 & 3.63 & 3.03 & 7.42 & 258.94 & 11.47 & 10.13 \\
\hline
\end{tabular}


A.M. Abd El-All, et al.,

\begin{tabular}{|c|c|c|c|c|c|c|c|}
\hline & Si & 3.73 & 3.12 & 7.89 & 265.44 & 11.83 & 9.99 \\
\cline { 2 - 8 } & Sw & 3.80 & 3.33 & 8.86 & 296.21 & 11.95 & 10.60 \\
\hline \multirow{3}{*}{ Cd300 } & 0 & 1.50 & 2.20 & 3.80 & 40.81 & 4.88 & 3.30 \\
\cline { 2 - 8 } & Si & 2.30 & 2.23 & 3.99 & 64.53 & 7.15 & 4.12 \\
\cline { 2 - 8 } & Sw & 2.75 & 2.75 & 6.09 & 180.90 & 9.20 & 8.70 \\
\hline \multicolumn{2}{|c|}{ LSD at 5\% } & 0.012 & 0.028 & 0.053 & 0.167 & 0.111 & 0.017 \\
\hline
\end{tabular}

Table (8b): Effect of heavy metals, nanoparticles and their interactions on yield and its components of tomato plants during the growing season of 2019.

\begin{tabular}{|c|c|c|c|c|c|c|c|}
\hline Treatments & Characters & $\begin{array}{l}\text { Fruit } \\
\text { length } \\
(\mathrm{cm})\end{array}$ & $\begin{array}{l}\text { Fruit } \\
\text { around } \\
\text { (cm) }\end{array}$ & $\begin{array}{c}\begin{array}{c}\text { Fruit } \\
\text { volume } \\
\left(\mathrm{cm}^{3}\right)\end{array}\end{array}$ & $\begin{array}{c}\text { Fruits } \\
\text { weight / } \\
\text { plant } \\
\text { (gm) }\end{array}$ & $\begin{array}{c}\text { Fruit } \\
\text { N. / } \\
\text { plant }\end{array}$ & $\begin{array}{c}\text { Vitamin C. } \\
\text { (mg/100g } \\
\text { f.wt. } \\
\text { fruits) }\end{array}$ \\
\hline $\begin{array}{l}\text { Heavy metals } \\
(\mathrm{ppm})\end{array}$ & $\begin{array}{c}\text { Inducers } \\
\text { (ppm) }\end{array}$ & \multicolumn{6}{|c|}{ Season II } \\
\hline 0 & - & 4.44 & 5.07 & 11.37 & 386.05 & 18.11 & 9.88 \\
\hline Pb1000 & - & 3.93 & 4.73 & 9.32 & 296.58 & 16.61 & 8.58 \\
\hline Pb2000 & - & 2.95 & 3.83 & 6.53 & 200.02 & 13.88 & 7.34 \\
\hline Cd100 & - & 3.55 & 4.15 & 7.68 & 231.72 & 15.53 & 7.50 \\
\hline $\mathrm{Cd} 300$ & - & 1.93 & 2.87 & 4.23 & 87.25 & 9.60 & 4.27 \\
\hline \multicolumn{2}{|c|}{ LSD at $5 \%$} & 0.532 & 0.154 & 0.709 & 35.160 & 1.079 & 0.047 \\
\hline- & 0 & 3.17 & 3.97 & 7.35 & 212.66 & 13.54 & 6.94 \\
\hline- & Si & 3.40 & 4.06 & 7.64 & 230.95 & 14.60 & 7.26 \\
\hline- & Sw & 3.51 & 4.37 & 8.49 & 277.36 & 16.10 & 8.33 \\
\hline \multicolumn{2}{|c|}{ LSD at $5 \%$} & 0.123 & 0.046 & 0.231 & 15.791 & 0.702 & 0.026 \\
\hline \multirow{3}{*}{0} & 0 & 4.28 & 4.94 & 11.06 & 339.20 & 16.77 & 9.16 \\
\hline & $\mathbf{S i}$ & 4.64 & 5.13 & 11.29 & 382.44 & 17.18 & 10.00 \\
\hline & Sw & 4.40 & 5.13 & 11.77 & 436.50 & 20.37 & 10.48 \\
\hline \multirow{3}{*}{ Pb1000 } & 0 & 3.75 & 4.69 & 8.89 & 283.46 & 15.91 & 8.53 \\
\hline & $\mathbf{S i}$ & 3.92 & 4.66 & 8.88 & 299.56 & 16.91 & 8.72 \\
\hline & Sw & 4.12 & 4.84 & 10.20 & 306.72 & 17.00 & 8.48 \\
\hline \multirow{3}{*}{ Pb2000 } & 0 & 2.86 & 3.71 & 6.33 & 181.29 & 13.34 & 6.91 \\
\hline & $\mathbf{S i}$ & 2.96 & 3.74 & 6.72 & 195.05 & 13.64 & 7.21 \\
\hline & Sw & 3.04 & 4.05 & 6.53 & 223.71 & 14.67 & 7.91 \\
\hline \multirow{3}{*}{ Cd100 } & 0 & 3.68 & 3.98 & 6.95 & 222.83 & 15.32 & 7.45 \\
\hline & $\mathbf{S i}$ & 3.50 & 4.12 & 7.60 & 217.33 & 15.85 & 6.99 \\
\hline & Sw & 3.46 & 4.34 & 8.48 & 254.99 & 15.41 & 8.05 \\
\hline \multirow{3}{*}{$\mathrm{Cd} 300$} & 0 & 1.27 & 2.52 & 3.50 & 36.51 & 6.37 & 2.67 \\
\hline & $\mathbf{S i}$ & 2.00 & 2.63 & 3.73 & 60.37 & 9.40 & 3.40 \\
\hline & Sw & 2.52 & 3.47 & 5.47 & 164.87 & 13.03 & 6.73 \\
\hline \multicolumn{2}{|c|}{ LSD at $5 \%$} & 0.018 & 0.023 & 0.069 & 0.139 & 0.093 & 0.021 \\
\hline
\end{tabular}

Conclusion 
It can be concluded from the present work that, lead and cadmium treatment, inhibited tomato plants growth, water relations and chemical Moreover, it was found that with increasing heavy metals concentrations, there was an increase in their concentrations in roots and shoots which reflected a negative effect on yield and its components, also increased antioxidants enzymes. The more interested obtained results that growing Eruca plants (hyperaccumulator) with tomato plants, resulted in highest accumulation of heavy metals in whole plants of watercress especially with increasing the level of heavy metal treatments. In addition, the ability of lead and cadmium accumulation in watercress plants having a positive correlation with seaweed and silicon application. The most effective treatment was seaweed which recorded a higher value of $\mathrm{Pb}$ and Cd accumulation. It can prove that silicon and seaweed treatments as foliar application on tomato plants enhanced plant tolerance to heavy metal toxicity. In addition, it is proved from this study that, the beneficial effect of growing hyperaccumulator plant like watercress with economic plants like tomato plants in form of intercropping system to prevent and protect tomato yield from the deleterious effects of heavy metals contamination with application silicon or seaweed in form of nanoparticls as foliar application to improve tomato tolerance to heavy metals and increasing watercress accumulation of heavy metal and reduce heavy metal uptake by tomato.

\section{REFERENCES}

Arun, D., P. Gayathri, K. Chandran and D. Yuvaraj (2014). Studies on Effect of Seaweed Extracts on Crop Plants and Microbes. International Journal of ChemTech Research. 6(9): 4235-4240.

A.O.A.C. (1995). Association of Official Agriculture Chemists. Official
Methods of Analysis. $16^{\text {th }}$ Ed. A.O.A.C. Virginia, DC, USA.

A. O. A. C. (1980). Official Methods of Analysis. $18^{\text {th }}$ ed. Association of Official Agricultural Chemists, Washington, DC, USA.

Barrs, H. D. and P. E. Weatherley (1962). Arc examination of the relative turgidity technique for estimating water deficits in leaves. Aust. J. Biol. Sci., 15: 413 - 428.

Broesh, S. (1954). Colorimetric assay of phenoloxidase. Bull. Soc. Chem., Biol., 36: 711-713.

Costat, Software (1985). Costat 6311Windowes by Roland Sneif, Internet.

Cottenie, A., M. Verloo, L. Kiekens, G. Velghe and R. Camerlyck (1982). Analytical Problems and methods in chemical plant and soil analysis. Hand book Ed.A.Cottenie, Gent, Belgium.

Dahlin, T. and M. H. Loke (1997). Quasi3D resistivity imaging:Mapping of 3D structures using two dimensional dc resistivitytechniques, 3rd Mtg., Environ. Eng. Geophys. Assn., ExpandedAbstracts, 143-146.

Dong, Q., J. Fang, F. Huang and Kunzheng (2019). Silicon Amendment Reduces Soil Cd Availability and Cd Uptake of Two Pennisetum Species. Int. J. Environ Res Public Health. 16(9): 1624.

Dubois, M., A. Gilles, K. J. Hamilton, P. R. Rebers and P.A. Smith (1956). Colorimetric method for determination of sugar and related substances. Anals. Chem., 28: 350.

El-Gamal, S. M. and S. AR. Hammad (2003). counteracting the deleterious effects of lead and cadmium on tomato plants by using yeast, garlic and eucalyptus extracts. Minufiya J. Agric. Res., 28(3): 737-755.

Etesamy, H. and B.R. Jeong (2018). Silicon (Si): Review and future 
prospects on the action mechanisms in alleviating biotic and abiotic stresses in plants. Ecotoxicology and Enviro. Safety.147: 881-896.

Ernst, W. H. O. (1996). Bioavailability of heavy metals and decontamination of soil by plants. Appl. Geochem., 11:163-167.

Fadeel, A. A. (1962). Location and properties of chloroplasts and pigment determination in shoots. Plant Physiol., 15: 130-137.

Fazal, H., B. Asghari and P. F. Michael (2010). The improved phytoextraction of lead $(\mathrm{Pb})$ and the growth of maize (Zea mays L.): the role of plant growth regulators (GA3 and IAA) and EDTA alone and in combinations. Chemosphere, 80: 457-462.

Fehrman, H. and A. E. Dimond (1967). Peroxidase activity and phytophthora resistance in different organs of the potato. Plant pathology, 57: 69-72.

Gomez, K. A. and A. A. Gomez (1984). Statistical procedures for agricultural research. $2^{\text {nd }}$ ed. Jahn Wiley Sons, New York, U.S.A. pp. 680.

Goyal, H., A. Bafna, T.R. Nagesh, N. Vyas and R. Gupta (2017). soluble silica as a boon for alleviating toxic effects of heavy metals on vigna radiata grown hydroponically in sewage. International Journal of Agricultural Science and Research (IJASR) 283294.

Giller, K. E., E. Witter and S. P. McGrath (1998). Toxicity of heavy metals to microorgan-isms and microbial processes in agricultural soils - a review, Soil Biol. Biochem. 30: 13891414.

Hashem, H., A. Showman and $R$. Hassanein (2017). Physicobiochemical properties of tomato (Solanum lycopersicum) grown in heavy-metal contaminated soil, https://doi.org/10.1080/09064710.2017.140 1113, 68: 334-341.

Hirsch, R. E., B. D. Lewis, E. P. Spalding and M. R. Sussman (1998). A role for the AKT1 potassium channel in plant nutrition. Science, 280(5365): 918-921.

Hussain, H., I., N. Kasinadhuni and T. Arioli (2021). The effect of seaweed extract on tomato plant growth, productivity and soil. Journal of Applied Phycology.33,1305-1314.

Khan, W., UP. Rayir ath and S. Subr amanian (2009). Seaweed extracts as biost imulants of plant growth and development. J. P lant Growth Regul., 28(4): 386-399.

Khan, Z., M. Rizwan and M. Hafeez (2020). Effects of silicon nanoparticles on growth and physiology of wheat in cadmium contaminated soil under different soil moisture levels. Environmental Science and pollution Research .27(14).

Kisa, D. (2018). The Responses of Antioxidant System against the Heavy Metal-Induced Stress in Tomato. Journal of Natural and Applied Sciences. 22(1) :1-6.

Li, M. Q., MK. Hasan, C. X. Li, GJ. Ahammed, J. Xia and K. Shi (2016). Melatonin mediates selenium-induced tolerance to cadmium stress in tomato plants. Journal of pineal Research. 61(3): 291-302.

Liu, W. X., J. W. Liu, M. Z. Wu, Y. Li, Y. Zhao and S. R Li (2009). Accumulation and translocation of toxic heavy metals in winter wheat (Triticum aestivum L.): Growing in Agricultural Soil of Zhengzhou, China. Bull Environ Contam. Toxicol., 82: 343-347.

Luptáková, A., D. Gešperová and D. Kupka (2002). The selective chemical biological precipitation of $\mathrm{Cu}$ and $\mathrm{Cd}$ from solutions, in.: Fečko, P., Čablík, V. (Eds.) Proceeding from 6th Conference on Environment and 
Mineral Processing, part II, Ostrava, 509-514.

Ma, J. F. (2004). Role of silicon in enhancing the resistance of plants to biotic and a biotic stress. Soil Sci. Plant Nutr., 50(1): 11-18.

Maksimovic, JD., M. Mojovic, V. Maksimovic, V. Romheld and $M$. Nikolic (2012). Silicon ameliorates manganese toxicity in cucumber by decreasing hydroxyl radical accumulation in the leaf apoplast. $J$ Exp Bot 63:2411-2420.

Page, A. L. (1982). Methods of Soil Analysis Part 2: Chemical and Microbiological Properties. Soil Sci. Amer. Inc., Madison, Wisconsin, USA.

Patel, D., T. R. Ahlawat, S. Jena and A. Chaudhary (2020). Effect of Silicon and Seaweed Extract on Physical and Sensory Quality of Papaya cv. Red Lady. Int. J. Curr. Microbiol. App. Sci. 9(1): 504-510.

Rieuwerts, J. S., M. Thornton, E. Farago and M. R. Ashmore (1998). Factors influencing metal bioavailability in soils: preliminary investigations for the development of a critical loads approach for metals. Chemical Speciation \& Bioavailability. 1(2).

Saad-Allah, KH. and A. Nessim (2016). Protective Role of the Seaweed Halimeda opuntia Extract on Cadmium-Stressed Eruca sativa (Mill). Egypt. J. Bot., 56 (3): 863-881.

Saeed, A., M. Sohail, N. Rashi and M. lqbal (2014). Effects of heavy metals toxicity on the biochemical response in tomato plants grown in contaminated silt-soil. Bangladesh J. Sci. and Ind. Res. 48(4): 229-236.

Salt, D. E., M. Blaylock, N. P. B. A. Kumar, V. Dushenkov, B. D. Ensley, I. Chet and I. Raskin (1995). Phytoremediation: A novel strategy for the removal of toxic metals from the environment using plants. Nature Biotechnology 13: 468-474.

Seregin, I. V. and V. B. Ivanov (2001). Physiological aspects of cadmium and lead toxic effects on higher plants. Russ J Plant Physiol., 48(4): 523-544.

Seregin, I. V. and A. D. Kozhevnikova (2008). Roles of root and shoot tissues in transport and accumulation of cadmium, lead, nickel, and strontium, Rus. J. Plant Physiol., 55: 1-22.

Sharma, P. and R. S. Dubey (2005). Lead toxicity in plants, Braz. J. Plant Physiol. 17: 35-52.

Sharma, N. C., S. V. Sahi and J. C. Jain (2006). Sesbania drummondii cell cultures: ICP-MS determination of the accumulation of $\mathrm{Pb}$ and $\mathrm{Cu}$. Microchemical Journal, 81: 163- 169.

Shi, X., C. Zhang, H. Wang and F. Zhang (2005). Effect of Si on the distribution of $\mathrm{Cd}$ in rice seedings. Plant Soil 272(1):53-60.

Shuyi, L., Z. Shirong, D. Xiaodong, L. Xinrong and W. Rongping (2013). Spraying Silicon or Cerium Sols Favorably Mediated Enhancement of $\mathrm{Cd}, \mathrm{Pb}$ Tolerance in Lettuce Grown in Combined $\mathrm{Cd}, \mathrm{Pb}$ Contaminated Soil. Procedia Environmental Sciences. 6877.

Singh, R. P. and M. Agrawal (2007). Effects of sewage sludge amendment on heavy metal accumulation and consequent responses of Beta vulgaris plants. Chemosphere, 67 (11): 2229-2240.

Sobkowiak, R. (2016). Water relations in plants subjected to heavy metal stresses. Acta Physiol Plant. 38: 257.

Sun, X., Q. Hu and L. Tan (2006). Effect of molybdenum on antioxidative defense system and membrane lipid peroxidation in winter wheat low temperature stress. Journal plant physiol. Mol. Biol., 2:175-18. 
Suriyaprabha, R., G. Karunakaran, R. Yuvakkumar, V. Rajendran and $\mathbf{N}$. Kannan (2012). Silica nanoparticles for increased silica availability in maize (Zea mays L.) seeds under hydroponic conditions. Curr Nano sci 8(6):902908.

Thirumaran, G., M. Arumugam, R. Arumugam and $P$. Anantharaman (2009). Effect of seaweed liquid fertilizer on growth and pigment concentration

of abelmoschusesculentus (I) medikus. American-Eurasian J. Agronomy 2 (2): 57-66.

Tripathi, D., V. Singh, P. Ahmad, D. Chauhan and S. Prasad (2016). Silicon and Cadmium Toxicity in Plants. In book, Silicon in Plants: Advances and Future Prospects. 245-246.

Tung, G. and P. J. Temple (1996). Uptake and localization of lead in corn (Zea mays L.) seedlings, a study by histochemical and electron microscopy. Sci Total Environ., 188(23): 71-85.

Verma, S. and R. S. Dubey (2003). Lead toxicity induces lipid peroxidation and alters the activities of antioxidant enzymes in growing rice plants. Plant Science, 164: 645-655.

Wang, H., X. Shan, B. Wen, G. Owens, J. Fang and S. Zhang (2007). Effect of indole-3-acetic acid on lead accumulation in maize (Zea mays L.) seedlings and the relevant antioxidant response. Environ Exp Bot., 61(3): 246-253.
Welch, R. M., W. A. Norvell, S. C. Schaefer, J. E. Shaff and L.V. Kochian (1993). Induction of iron (III) and copper (II) reduction in pea (Pisum sativum $\mathrm{L}$.) roots by $\mathrm{Fe}$ and $\mathrm{Cu}$ status: does the root-cell plasmalemma $\mathrm{Fe}$ (III)-chelate reductase perform a general role in regulating cation uptake. Planta, 190: 555-561.

Wu, J., J. Guo, Y. Hu and H. Gong (2015). Distinct physiological responses of tomato and cucumber plants of silicon-mediated alleviation of cadmium stress. Frontiers in plant science. 6; 453.

Yilmaz, D. D. and K. U. Parlak (2011). Changes in proline accumulation and antioxidative enzyme activities in Groenlandia densa under cadmium stress. Ecological Indicators, 11: 417423.

Yongsheng, W., Q. Liang and Q. Tang (2011). Effect of $\mathrm{Pb}$ on growth, accumulation and quality component of tea plant. Procedia Engineering, 18: $214-219$.

Zargar, S. M., R. Mahajan, J. Bhat, M. Nazir and R. Deshmukh (2019). Role of silicon in plant stress tolerance: opportunities to achieve a sustainable cropping system. Biotech, 9(3): 2-16.

Zodape, S., S. Mukherjee, M. Reddy and D. Chaudhary (2010). Effect of Kappaphycus alvarezii (Doty) Doty ex silva. extract on grainquality, yield and some yield components of wheat (Triticumaestivum L.), Int $\mathbf{J}$ Plant Prod.,3 : 97-101. 


\title{
دراسات فسيولوجية على آليات مقاومة المعادن الثقيلة في نمو نباتات الطماطم تحت ظروف التحميل
}

\author{
أحمد محمد عبدالعال ، إسراء علي مرسي ، مرفت إدوارد سوريال

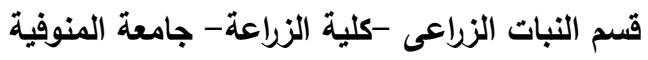

الملخص العربى

أجريت تجارب أصص في صوية كلية الزراعة بشبين الكوم جامعة المنوفية خلال موسي

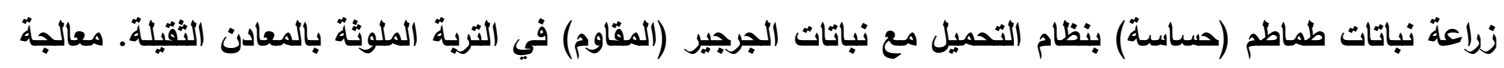

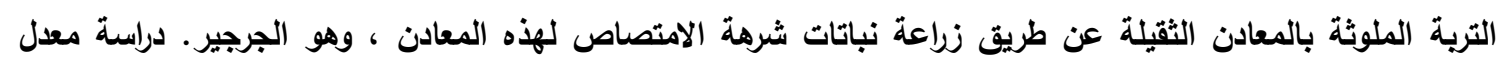

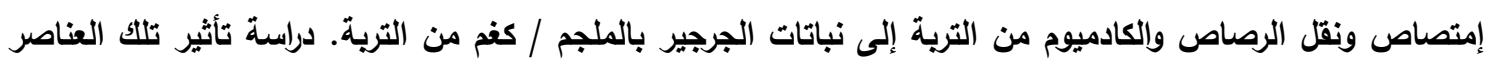
الثقيلة على النمو والعلاقات المائية والمحتوى الكيميائي وكذلك المحصول ومكوناته لنباتات الطماطم. تم إستخدام ثثلاثة مستويات من الرصاص وهي صفر (كنترول) و 1000 و 2000 ملجم / كجم من التربة وثلاثة مستويات من الكادميوم وهي صفر (كنترول) و 100 و 300 ملجم / كجم من التربة كل منها على حدة. مع إستخدام محفزات النمو مثل جزيئات

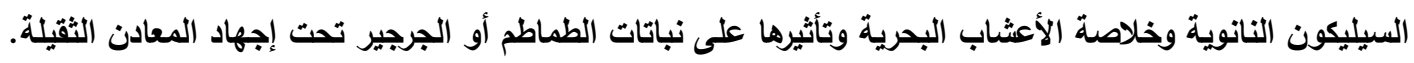

تمت دراسة خصائص النمو الخضري ويعض الخصائص الفسيولوجية والكيميائية مثل أصباغ التمثيل الضوئي والعلاقات المائية ونثاط بعض الإنزيمات المضادة للأكسدة وتراكم الرصاص والكادميوم ويعض الخصان الثصائص الكمية والنوعية

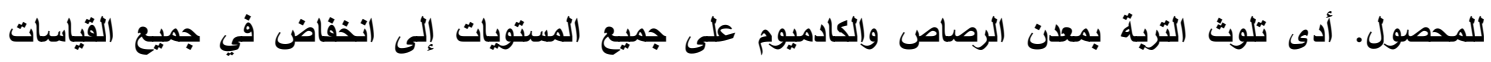

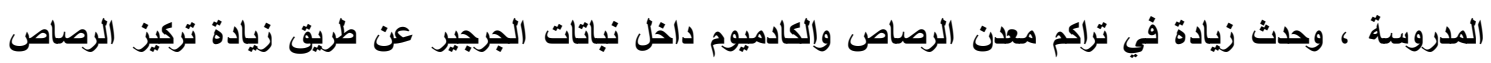

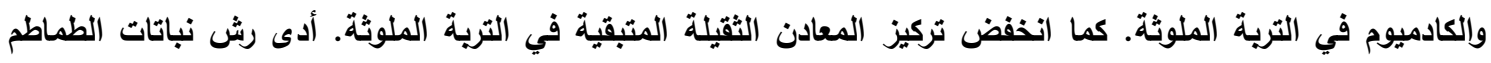

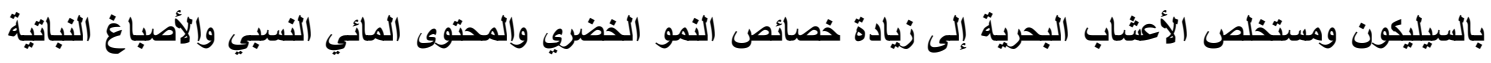

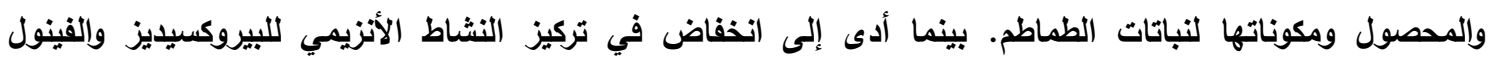

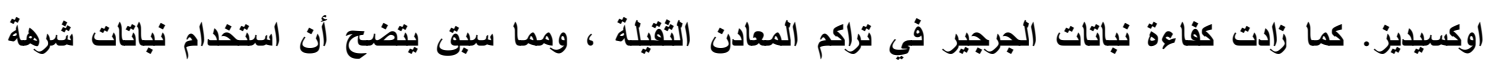

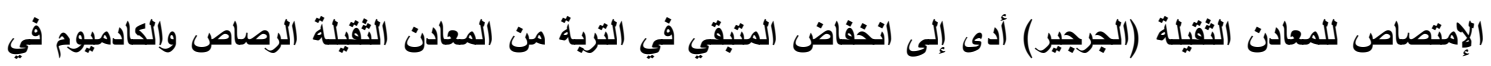

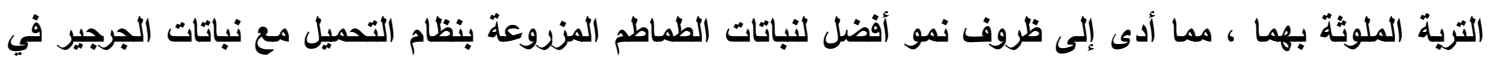

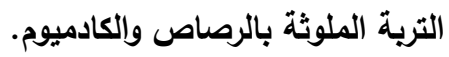


أسماء السادة المحكمين

أ.د/ محمد أحمد محمد ماضى كلية الزراعة - جامعة بنها

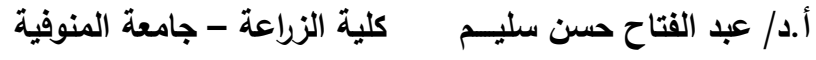

